\title{
Study of helicity properties of peculiar active regions
}

\author{
M. C. López Fuentes ${ }^{1}$, C. H. Mandrini ${ }^{1}$ and P. Démoulin ${ }^{2}$ \\ ${ }^{1}$ Instituto de Astronomía y Física del Espacio (CONICET-UBA), CC 67, Suc 28, 1428 Buenos \\ Aires, Argentina \\ e-mail: lopezf@iafe.uba.ar \\ ${ }^{2}$ Observatoire de Paris, LESIA, UMR 8109 (CNRS), F-92195, Meudon Principal Cedex,
}

France

\begin{abstract}
Peculiar solar active regions (ARs), such as $\delta$-islands and other high tilt bipoles, are commonly associated with the emergence of severely deformed magnetic flux tubes. Therefore, the study of these ARs provides valuable information on the origin and evolution of magnetic structures in the solar interior. Here, we infer the magnetic helicity properties of the flux tubes associated to a set of peculiar ARs by studying the evolution of photospheric magnetograms (SOHO/MDI) and coronal observations (SOHO/EIT and TRACE) in combination with forcefree models of the magnetic field. We discuss how our results relate to different models of the evolution of emerging magnetic flux tubes.
\end{abstract}

Keywords. Sun: magnetic fields, Sun: activity, Sun: photosphere, Sun: corona

\section{Introduction}

Solar active regions (ARs) are the manifestation of the buoyant emergence of magnetic flux tubes from the solar interior. Since these magnetic structures are related to the toroidal field at the base of the convection zone, they are approximately parallel to the solar equator. For this reason, most ARs are bipolar configurations oriented in the eastwest direction, obeying what is known as Hale's law (e.g., Schrijver \& Swaan 2000). AR bipoles are generally slightly inclined, so the preceding polarities tend to be closer to the solar equator (Joy's law). This inclination ("tilt" angle) is thought to be due to the Coriolis force acting on the flux tubes during emergence (see Fisher et al. 2000). A number of ARs have tilts that deviates from Hale's and Joy's laws. A typical case are the " $\delta$-islands"; sunspots formed by two or more umbrae of different polarities within the same penumbra. These regions are associated to flux tubes that suffered a deformation with respect to the normal " $\Omega$ " loop shape. Among the possible causes of the deformation are the interaction of the flux tubes with the convection zone plasma and the development of the kink instability (López Fuentes et al. 2003, Tian et al. 2005). The deformation of the flux tube axis (the tube as a whole) is associated to the magnetic helicity component named writhe. The other component, called twist, corresponds to the rotation of the individual magnetic field lines around the main axis of the tubes. It is expected that, as the flux tube emerges through the photosphere, the peculiar AR bipole rotates towards the normal east-west direction. Therefore, from the rotation of the polarities during flux emergence we can infer the left (negative writhe) or right (positive writhe) chirality of the tubes. The sign of the flux-tube twist, on the other hand, can be inferred comparing force-free models of the coronal magnetic field with EUV and soft X-ray observations. The relation between writhe and twist provides information on the mechanism that produces the flux tube deformation. The kink instability, for instance, implies that both 

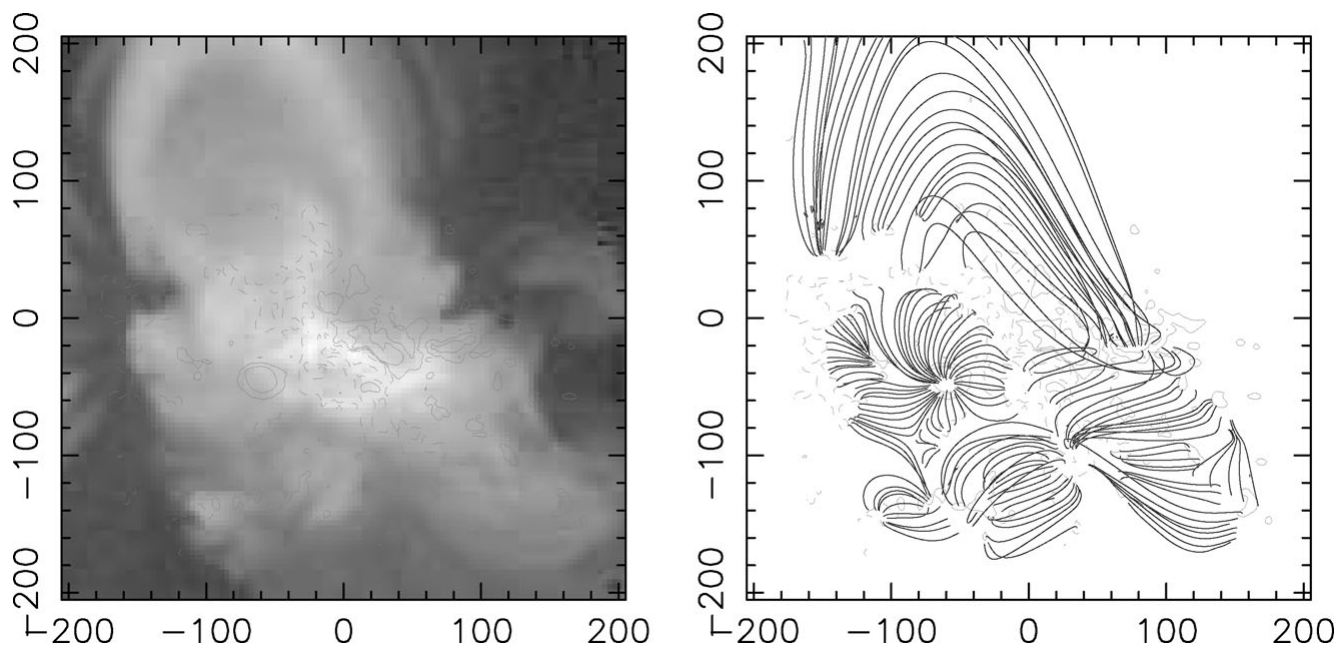

Figure 1. Left panel: Yohkoh/SXT image of AR 9026 observed on 2000 June 7 (14:52UT). Right panel: linear force-free magnetic model of the AR's coronal field extrapolated from SOHO/MDI magnetogram data. Continuous/dashed contours correspond to positive/negative longitudinal component of the photospheric magnetic field of levels \pm 100 and \pm 500 Gauss.

components must have the same sign, because it is due to the internal transfer of magnetic helicity from twist to writhe (Fan et al. 1999). Here, we analyze a set of peculiar ARs and we obtain the twist and writhe signs of the associated magnetic flux tubes to constrain the possible mechanisms at the origin of their deformation.

\section{Analysis and results}

We study 4 peculiar ARs from a larger set presented in López Fuentes \& Mandrini (2008). Using SOHO/MDI magnetograms we compute the mean position of the positive and negative polarities from which we obtain the tilt angle. From the tilt rotation during the AR evolution across the solar disk we infer the writhe of the associated magnetic flux tubes. It can be demonstrated that counterclockwise rotation (CCW) of the polarities corresponds to positive writhe, while clockwise $(\mathrm{CW})$ rotation indicates a negative writhe. In Table 1 we show the properties of the studied ARs. The first 5 columns correspond to: AR number, AR type, solar hemisphere to which the AR belongs, sense of rotation of the tilt during evolution, and inferred writhe sign.

To infer the sign of the AR twist we compare EUV and soft X-ray observations with models of the coronal magnetic field extrapolated from MDI magnetograms using the linear force-free equation:

$$
\nabla \times \mathbf{B}=\alpha \mathbf{B}
$$

We use the sign of the parameter $\alpha$ as a proxy for the twist sign. Figure 1, left panel, shows a soft X-ray image of AR 9026 obtained on 2000 June 7 with Yohkoh/SXT. In the right panel we show a model with $\alpha=-0.006 \mathrm{Mm}^{-1}$ extrapolated from a SOHO/MDI magnetogram obtained on 2000 June 7 (11:12UT). The comparison clearly shows that the AR has negative twist. For a more quantitative determination of the twist, we compute the mean distance of model field lines to a series of coronal loops from TRACE and Yohkoh/SXT. The model that best approaches the observed loops is considered the best fitting for the AR twist. In Figure 2 we plot the mean field line distance to TRACE loops versus the alpha parameter for AR 9026 (left panel) and AR 10808 (right panel). 

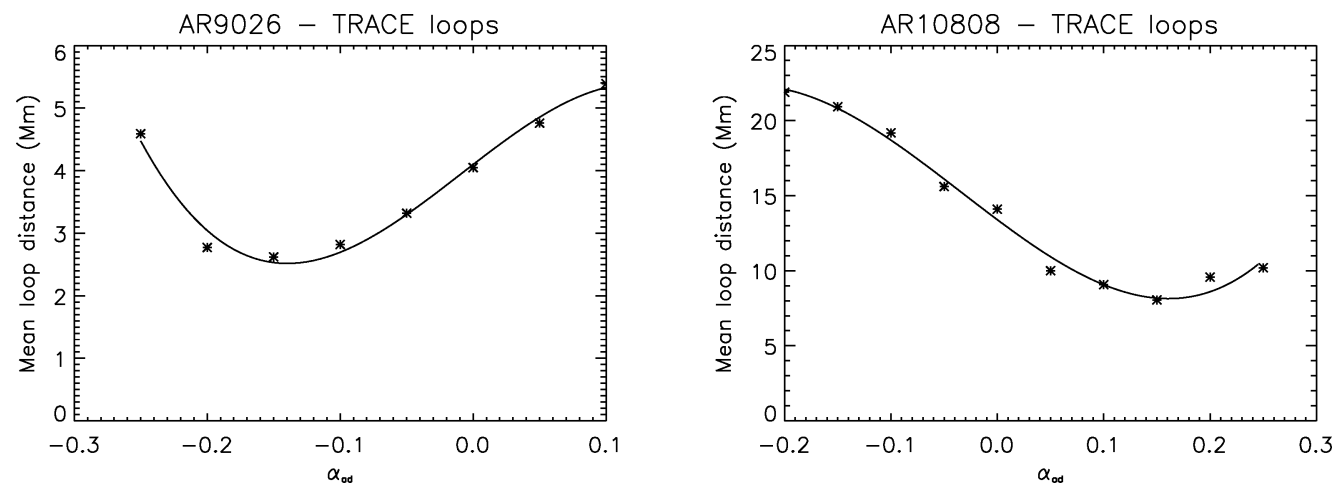

Figure 2. Mean distance between modeled magnetic field lines and observed coronal loops versus the force free parameter $\alpha$ for ARs 9026 and 10808. Curves are polynomial fittings.

Table 1. Properties of the studied ARs.

\begin{tabular}{ccccccc}
\hline AR Number & AR Type & Hem. & Rotation & W sign & T sign & Kink? \\
\hline 9026 & $\delta$-island & N & CW & Neg & Neg & $\sqrt{ }$ \\
9165 & High tilt & N & CW & Neg & Neg & $\sqrt{ }$ \\
9591 & Non-Hale & S & CCW & Pos & Neg & - \\
10808 & $\delta$-island & S & CCW & Pos & Pos & $\sqrt{ }$ \\
\hline
\end{tabular}

The fitting procedure shows that AR 9026 has negative twist and AR 10808 has positive twist. Similar analysis for AR 9165 and AR 9591 indicate that both ARs have negative twist (see Table 1). The last column of the table indicates whether the relation between twist and writhe signs is consistent with the kink instability or not.

\section{Conclusion}

We determine the magnetic helicity properties (twist and writhe) of 4 peculiar active regions that depart appreciably from Hale's law. Our main goal is to constrain the mechanisms proposed to explain magnetic flux-tube deformations. According to their twist and writhe signs, 3 of the studied cases are consistent with the development of a kink instability. Other mechanism, such as the interaction with the convection zone plasma during emergence, should be invoked to explain the remaining case.

\section{References}

Fan, Y., Zweibel, E. G., Linton, M. G., \& Fisher, G. H. 1999, ApJ, 521, 460

Fisher, G. H., Fan, Y., Longcope, D. W., Linton, M. G., \& Pevtsov, A. A. 2000, Solar Phys., 192, 119

López Fuentes, M. C. \& Mandrini, C. H. 2008, Boletín de la Asociación Argentina de Astronomía La Plata Argentina, 51, 31

López Fuentes, M. C., Démoulin, P., Mandrini, C. H., Pevtsov, A. A., \& van Driel-Gesztelyi, L. 2003, A\&A, 397, 305

Schrijver, C. J. \& Zwaan, C. 2000, Solar and stellar magnetic activity/Carolus J. Schrijver, Cornelius Zwaan. New York: Cambridge University Press, 2000. (Cambridge astrophysics series; 34)

Tian, L., Alexander, D., Liu, Y., \& Yang, J. 2005, Solar Phys., 229, 63 